\title{
PROPER HOLOMORPHIC MAPPINGS IN SOME CLASS OF UNBOUNDED DOMAINS
}

\author{
Armen Edigarian AND WlodZIMIERZ ZwONEK
}

\begin{abstract}
In the paper the problem of the form of proper holomorphic mappings between elementary Reinhardt domains of the rational type in $C^{2}$ is solved. The pluricomplex Green function with many poles is used in the solution of the problem. Additionally, we solve the holomorphic equivalence problem of elementary Reinhardt domains of the arbitrary type in $\boldsymbol{C}^{n}$ The results in the paper are generalizations of those from two papers of S. Shimizu.
\end{abstract}

Let $D$ be a domain in $C^{n}$. Fix a finite set $\emptyset \neq P \subset D$ and a function $v: P \rightarrow(0, \infty)$. Denote by $\mathscr{K}_{D, P, v}$ the family of functions satisfying the following conditions:

$u \in \operatorname{PSH}(D), u<0$ and for any $p \in P, u(z)-v(p) \log \|z-p\|$

is bounded above for $z$ near $p$

(we allow a plurisubharmanic function to be identically $-\infty$ ). Let us define the pluricomplex Green function with poles in $P$ and the weights $v$ (see [Lel]) as follows:

$$
g_{D}(P ; v ; w):=\sup \left\{u(w): u \in \mathscr{K}_{D, P, v}\right\}, \quad w \in D .
$$

If $v \equiv 1$, then we denote $g_{D}(P ; w):=g_{D}(P ; v ; w)$. In the special case $P=\{p\}$, $v(p)=1$ we denote $g_{D}(p ; w):=g_{D}(P ; v ; w)$ - it is a pluricomplex Green function with logarithmic pole at $p$ defined by M. Klimek (see [Kli]).

The following inequalities are easy to verify:

$$
\min \left\{v(p) g_{D}(p ; w): p \in P\right\} \geq g_{D}(P ; v ; w) \geq \sum_{p \in P} v(p) g_{D}(p ; w) .
$$

It is also well-known that $g_{D}(P ; v ; \cdot) \in \mathscr{K}_{D, P, v}$.

Put $V_{0}:=\left\{z=\left(z_{1}, \ldots, z_{n}\right) \in \boldsymbol{C}^{n}: z_{1} \cdots z_{n}=0\right\}$. Define

Let $\alpha=\left(\alpha_{1}, \ldots, \alpha_{n}\right) \in\left(\boldsymbol{R}_{+}\right)^{n}$, where $n>1\left(\boldsymbol{R}_{+}\right.$stands for positive numbers).

$$
D_{\alpha}:=\left\{z=\left(z_{1}, \ldots, z_{n}\right) \in C^{n}:\left|z_{1}\right|^{\alpha_{1}} \cdots\left|z_{n}\right|^{\alpha_{n}}<1\right\} .
$$

Research partially supported by the KBN grant No. 2 PO3A 06008 .

Received August 20, 1997; revised April 28, 1999. 
The domains $D_{\alpha}$ are called elementary Reinhardt domains.

For $w \in D_{\alpha}$, denote $\mathscr{J}(w):=\left\{j \in\{1, \ldots, n\}: w_{j}=0\right\}=\left\{j_{1}(w), \ldots, j_{k}(w)\right\}$.

Put $\tilde{D}_{\alpha}:=\left\{z \in D_{\alpha}: z_{1} \cdots z_{n} \neq 0\right\}=D_{\alpha} \backslash V_{0}$.

Let $\alpha$ be of rational type, i.e. $\alpha=\left(\beta \alpha_{1}, \ldots, \beta \alpha_{n}\right)$, where $\beta>0$ and $\alpha_{j}$ 's are relatively prime natural numbers. In this case we always assume $\beta=1$, and for any $\mu \in E:=\{\lambda \in C:|\lambda|<1\}$ define

$$
V_{\mu}^{\alpha}:=\left\{z \in D_{\alpha}: z^{\alpha}=\mu\right\}
$$

where $z^{\alpha}:=z_{1}^{\alpha_{1}} \cdots z_{n}^{\alpha_{n}}$. Note that $V_{0}^{\alpha}=V_{0}$.

For $w \in D_{\alpha}$ and $\alpha$ of rational type put $r_{\alpha}(w):=\operatorname{ord}_{w}\left(z^{\alpha}-w^{\alpha}\right)$. Note that

$$
\left.u\right|_{V_{\mu}^{\alpha}} \equiv \text { const. }
$$

for any bounded above plurisubharmonic function $u$ on $D_{\alpha}$.

The theory of the Green function with many poles suffers from lack of examples of domains for which the effective formulas of the Green function are known. We give below formulas for the function in the domains that we have just defined. The formulas for the Green function of elementary Reinhardt domains with one pole were obtained in [Jar-Pfl] (in the rational case) and in [Pfl-Zwo] (in the irrational case). Let us recall these formulas.

TheOREM 1 (see [Jar-Pfl], [Pfl-Zwo]). If $\alpha$ is of rational type, then

If $\alpha$ is of irrational type, then

$$
g_{D_{\alpha}}(p ; w)=\left(1 / r_{\alpha}(p)\right) \log \left|\frac{w^{\alpha}-p^{\alpha}}{1-\overline{p^{\alpha}} w^{\alpha}}\right| .
$$

$$
g_{D_{\alpha}}(p ; w)= \begin{cases}-\infty & \text { if } p \in \tilde{D}_{\alpha} \\ \frac{1}{\alpha_{J_{1}(p)}+\cdots+\alpha_{J_{k}(p)}} \log \left(\left|w_{1}\right|^{\alpha_{1}} \cdots\left|w_{n}\right|^{\alpha_{n}}\right), & \text { if } p \in V_{0} \cap D_{\alpha} .\end{cases}
$$

In our paper, utilizing the formulas from Theorem 1 we obtain the formulas for the Green function in elementary Reinhardt domains with many poles.

THEOREM 2. Let $\alpha$ be of rational type. Then the following formula holds:

$$
g_{D_{\alpha}}(P ; v ; w)=\sum_{\mu \in E: P \cap V_{\mu}^{\alpha} \neq \emptyset}\left(\max _{p \in V_{\mu}^{\alpha} \cap P}\left(v(p) / r_{\alpha}(p)\right)\right) \log \left|\frac{w^{\alpha}-\mu}{1-\bar{\mu} w^{\alpha}}\right| .
$$

If $\alpha$ is of irrational type then

$$
\begin{aligned}
& g_{D_{\alpha}}(P ; v ; w) \\
& \quad= \begin{cases}-\infty, & \text { if } P \cap \tilde{D}_{\alpha} \neq \emptyset \\
\max _{p \in P}\left(\frac{v(p)}{\alpha_{J_{1}(p)}+\cdots+\alpha_{J_{k}(p)}}\right) \log \left(\left|w_{1}\right|^{\alpha_{1}} \cdots\left|w_{n}\right|^{\alpha_{n}}\right), & \text { if } P \cap \tilde{D}_{\alpha}=\emptyset .\end{cases}
\end{aligned}
$$


Let $\pi: \tilde{D} \rightarrow D$ be a proper holomorphic mapping (with multiplicity $m$ ) and let $P=:\left\{p_{1}, \ldots, p_{N}\right\}$ be a set of poles in $D$ such that $\pi^{-1}(P) \cap\left\{\operatorname{det} \pi^{\prime}=0\right\}=\emptyset$ and let $v_{j}:=v\left(p_{j}\right), j=1, \ldots, N$. Define

$$
\pi^{-1}(P ; v):=\bigcup_{j=1}^{N}\left\{\left(p_{j}^{k}, v_{j}\right): k=1, \ldots, m\right\},
$$

where $\pi^{-1}\left(p_{j}\right)=\left\{p_{j}^{1}, \ldots, p_{j}^{m}\right\}$ for $j=1, \ldots, N$. We shall use the following theorem, whose proof is to be found in [Lár-Sig] and [Edi-Zwo].

THEOREM 3. Under the assumptions as above, for any $\tilde{w} \in \tilde{D}$ the following formula holds:

$$
g_{\tilde{D}}\left(\pi^{-1}(P ; v) ; \tilde{w}\right)=g_{D}(P ; v ; \pi(\tilde{w})) .
$$

In [Shi1] and [Shi2] the problem of holomorphic equivalence of domains $D_{\alpha}$ was considered. Below we shall extend the results from the first of Shimizu's paper to proper mappings between the domains of the rational type and we will give a full description of such mappings (still in $C^{2}$ ). We also extend the results of [Shi2] (the solution of the holomorphic equivalence problem of elementary Reinhardt domains in $C^{2}$ ) to an arbitrary dimension. To be more precise, using Theorem 3 we shall prove the following result:

THEOREM 4. Let $F: D_{\alpha} \mapsto D_{\beta}$, where both $\alpha$ and $\beta$ are of rational type and $n=2$, be a proper holomorphic mapping. Then $F$ is one of the following forms:

$$
\left\{\begin{array}{l}
F\left(z_{1}, z_{2}\right)=\left(a^{1 / \beta_{1}}\left(z_{1}^{\alpha_{1}} z_{2}^{\alpha_{2}}\right) z_{1}^{k_{1}}, e^{i \theta} z_{2}^{k_{2}} / a^{1 / \beta_{2}}\left(z_{1}^{\alpha_{1}} z_{2}^{\alpha_{2}}\right)\right) \text { or } \\
F\left(z_{1}, z_{2}\right)=\left(a^{1 / \beta_{1}}\left(z_{1}^{\alpha_{1}} z_{2}^{\alpha_{2}}\right) z_{2}^{l_{1}}, e^{i \theta} z_{1}^{l_{2}} / a^{1 / \beta_{2}}\left(z_{1}^{\alpha_{1}} z_{2}^{\alpha_{2}}\right)\right),
\end{array}\right.
$$

where $a$ is any holomorphic function defined on $E$ omitting $0, \theta \in \boldsymbol{R}, k_{1}, k_{2}, l_{1}, l_{2}$ are positive integers satisfying the following relations:

$$
\alpha_{2} \beta_{1} k_{1}=\alpha_{1} \beta_{2} k_{2}, \alpha_{1} \beta_{1} l_{1}=\alpha_{2} \beta_{2} l_{2} .
$$

Our next result states that two domains under consideration (in arbitrary dimension) are biholomorphic only when they are linearly biholomorphic. As already mentioned, this result extends the theorem from [Shi2] (where the same result, but with other methods, was obtained in dimension 2).

THEOREM 5. If $F: D_{\alpha} \mapsto D_{\beta}$ is a biholomorphic mapping, then there is a permutation $\sigma$ of $\{1, \ldots, n\}$ and $t>0$ such that $\alpha_{j}=t \beta_{\sigma(j)}$.

Proof of Theorem 2. Note that the functions on the right hand-sides of the formulas in Theorem 2 are from $\mathscr{K}_{D_{x}, P, v}$, which gives us the inequalities ' $\geq$ '.

Consider now the rational case. Define $\tilde{P}$ to be a subset of $P$ such that all 
$q^{\alpha}(q \in \tilde{P})$ are different and for any $q \in \tilde{P}$ the following relation holds:

$$
\frac{v(q)}{r_{\alpha}(q)}=\max \left\{\frac{v(p)}{r_{\alpha}(p)}: p^{\alpha}=q^{\alpha}, p \in P\right\} .
$$

Define $\tilde{v}: \tilde{P} \mapsto(0, \infty)$ by $\tilde{v}:=\left.v\right|_{\tilde{P}}$. Note that because of $(2)$ we have that

$$
u \in \mathscr{K}_{D_{\alpha}, P, v} \quad \text { iff } u \in \mathscr{K}_{D_{\alpha}, \tilde{P}, \tilde{v}} .
$$

Consequently,

$$
g_{D_{\alpha}}(P ; v ; \cdot)=g_{D_{\alpha}}(\tilde{P} ; \tilde{v} ; \cdot) .
$$

This allows us to consider only such $P$ that for all $p \in P$ the numbers $p^{\alpha}$ are different. Now proceeding exactly as in [Jar-Pfl] we may prove for any $u \in$ $\mathscr{K}_{D_{\alpha}, P, v}$ there is $v \in \mathscr{K}_{E, Q, \mu}$, where $Q:=\left\{p^{\alpha}: p \in P\right\}, \mu\left(p^{\alpha}\right):=v(p) / r_{\alpha}(p)$ such that $v\left(z^{\alpha}\right)=u(z)$. This implies that $g_{D_{\alpha}}(P ; v ; z) \leq g_{E}\left(Q, \mu, z^{\alpha}\right)$, which finishes the case (use the formula for $\left.g_{E}\left(Q ; \mu ; z^{\alpha}\right)\right)$.

In the irrational case note that the desired formula equals $\min _{p \in P}\left\{v(p) g_{D_{\alpha}}(p ; w)\right\}$ (see [Pfl-Zwo]). Therefore, the first inequality in (1) finishes the proof.

We show the results announced with the help of two Lemmas. In Lemmas 6 and 7 we consider only domains of rational type.

Lemma 6. Let $\alpha$ and $\beta$ be of rational type. If $F: D_{\alpha} \mapsto D_{\beta}$ is holomorphic, then for any $\lambda \in E$ there is $\mu \in E$ such that

$$
F\left(V_{\lambda}^{\alpha}\right) \subset V_{\mu}^{\beta} .
$$

Proof. In fact, we know that (see Theorem 1)

$$
\begin{aligned}
\log \left|\frac{w^{\alpha}-z^{\alpha}}{1-\overline{w^{\alpha}} z^{\alpha}}\right|^{1 / r_{\alpha}(w)} & =g_{D_{\alpha}}(w ; z) \\
& \geq g_{D_{\beta}}(F(w) ; F(z)) \\
& =\log \left|\frac{(F(w))^{\beta}-(F(z))^{\beta}}{1-\overline{(F(w))^{\beta}}(F(z))^{\beta}}\right|^{1 / r_{\beta}(F(w))}, \quad w, z \in D_{\alpha} .
\end{aligned}
$$

Consequently, if $w^{\alpha}=z^{\alpha}$, then $(F(w))^{\beta}=(F(z))^{\beta}$ and we get (3).

In view of Lemma 6 we may define a mapping $\varphi: E \mapsto E$ by the equality $\varphi(\lambda):=\mu$.

What are the regularity properties of $\varphi$ under the assumption that $F$ is holomorphic? First, note that $\varphi$ is continuous on $E$.

For the proof of the fact that $\varphi$ is holomorphic take any $\lambda_{0} \neq 0$. Consider now $\lambda$ close to $\lambda_{0}$. Then taking a holomorphic root we have that $\varphi(\lambda)=$ 
$\left(F_{1}\left(1, \ldots, 1, \lambda^{1 / \alpha_{n}}\right)\right)^{\beta_{1}} \cdots\left(F_{n}\left(1, \ldots, 1, \lambda^{1 / \alpha_{n}}\right)\right)^{\beta_{n}}$ is holomorphic for $\lambda$ close enough to $\lambda_{0}$. This implies (together with the continuity) that $\varphi$ is holomorphic on $E$.

Our next lemma is the following

Lemma 7. If $F: D_{\alpha} \mapsto D_{\beta}(\alpha$ and $\beta$ are of the rational type) is a proper holomorphic mapping, then $\varphi$ is proper. In particular, there are $\lambda_{1}, \ldots, \lambda_{k} \in E$ and $\theta \in \boldsymbol{R}$ such that $\varphi(\lambda)=e^{i \theta} \prod_{\jmath=1}^{k}\left(\lambda-\lambda_{\jmath}\right) /(1-\bar{\lambda}, \lambda), \lambda \in E$.

Proof. To prove the lemma take any $z \in D_{\alpha}$. Take any $w \in \tilde{D}_{\alpha}$ such that $F(w) \notin F\left(\left\{\operatorname{det} F^{\prime}=0\right\}\right.$ ) (such a $w$ obviously exists because of the properties of proper holomorphic mappings). Then Theorem 3 together with the formulas from Theorem 2 gives us

$$
\sum_{\lambda: \varphi(\lambda)=(F(w))^{\beta}}\left(\max _{\tilde{w}^{\alpha}=\lambda, F(\tilde{w})=F(w)} \frac{1}{r_{\alpha}(\tilde{w})}\right) \log \left|\frac{\lambda-z^{\alpha}}{1-\bar{\lambda} z^{\alpha}}\right|=\log \left|\frac{(F(w))^{\beta}-(F(z))^{\beta}}{1-\overline{(F(w))^{\beta}}(F(z))^{\beta}}\right| .
$$

Taking now $\left|z^{\alpha}\right| \rightarrow 1$ we get that $\left|F(z)^{\beta}\right| \rightarrow 1$, which finishes the proof.

Now we can go on to the proof of Theorem 4 .

Proof of Theorem 4. At first we consider the very special case $\alpha=\beta=$ $(1,1)$. Note that the mappings

$$
\boldsymbol{C}_{*} \ni z \mapsto F_{J}\left(z, \frac{\lambda}{z}\right) \in \boldsymbol{C}_{*}, \quad \lambda \in E \backslash\left\{\lambda_{1}, \ldots, \lambda_{k}, 0\right\}, \quad j=1,2
$$

are holomorphic and proper.

Consequently, because of the form of proper holomorphic self-mappings of $C_{*}$ we get that

$$
F_{J}\left(z, \frac{\lambda}{z}\right)=a_{j}(\lambda) z^{r_{J}(\lambda)}, \quad \lambda \in E \backslash\left\{\lambda_{1}, \ldots, \lambda_{k}, 0\right\}, \quad j=1,2, \quad z \in C_{*},
$$

where $a_{j} \in \mathcal{O}\left(E \backslash\left\{\lambda_{1}, \ldots, \lambda_{k}, 0\right\}, \boldsymbol{C}_{*}\right)\left(a_{j}(\lambda):=F_{j}(1, \lambda / 1)\right), \quad r_{j}(\lambda) \in \boldsymbol{Z} \backslash\{0\}, j=1,2$, $\lambda \in E \backslash\left\{\lambda_{1}, \ldots, \lambda_{k}, 0\right\}$. It is easy to see that there is an $r_{j}$ such that $r_{j}(\lambda)=r_{j}$ for any $\lambda \in E \backslash\left\{\lambda_{1}, \ldots, \lambda_{k}, 0\right\}$ (use the continuity of the mappings). Moreover, $a_{j}$ is holomorphic on $E$ (but it may happen that $a_{j}\left(\lambda_{l}\right)=0$ ).

First we shall prove that

$$
\lambda_{1}=\cdots=\lambda_{k}=0 .
$$

Suppose that it does not hold. Without loss of generality we may assume that $a_{1}\left(\lambda_{1}\right)=0$ and $\lambda_{1} \neq 0$. Then by the properness of $F$ the mapping $\psi(z):=$ $F_{2}\left(z, \lambda_{1} / z\right): \boldsymbol{C}_{*} \rightarrow \boldsymbol{C}$ is proper. On the other hand we know that $\psi(z)=$ $a_{2}\left(\lambda_{1}\right) z^{r_{2}}$ - contradiction.

From Lemma 7 together with (4) we get $a_{1}(\lambda) z^{r_{1}} a_{2}(\lambda) z^{r_{2}}=e^{i \theta} \lambda^{k}$, so $r_{1}+$ $r_{2}=0$, (write $r:=r_{1}=-r_{2}$ ) and $a_{2}(\lambda)=e^{i \theta} \lambda^{k} / a_{1}(\lambda)$. Consequently, we have 
(take $\left.z:=z_{1}, \lambda:=z_{1} z_{2}\right)$

$$
F_{1}\left(z_{1}, z_{2}\right)=a_{1}\left(z_{1} z_{2}\right) z_{1}^{r}, \quad F_{2}\left(z_{1}, z_{2}\right)=\left(e^{i \theta} z_{1}^{k} z_{2}^{k}\right) /\left(z_{1}^{r} a_{1}\left(z_{1} z_{2}\right)\right) .
$$

We may write $a_{1}(\lambda)=a(\lambda) \lambda^{t}$, where $a \in \mathcal{O}\left(E, C_{*}\right), t \geq 0, t \in Z$, so

$$
F\left(z_{1}, z_{2}\right)=\left(a\left(z_{1} z_{2}\right) z_{1}^{r+t} z_{2}^{t}, e^{i \theta} z_{1}^{k-r-t} z_{2}^{k-t} / a\left(z_{1} z_{2}\right)\right) .
$$

Certainly it must hold $r+t \geq 0, k-t \geq 0$ and $k-t-r \geq 0$. Properness property may be preserved only if $(r+t=0$ and $k-t=0)$ or $(t=0$ and $k-r-t=0$ ), which gives us two possibilities

$$
\left\{\begin{array}{l}
F\left(z_{1}, z_{2}\right)=\left(a\left(z_{1} z_{2}\right) z_{1}^{k}, e^{i \theta} z_{2}^{k} / a\left(z_{1} z_{2}\right)\right) \text { or } \\
F\left(z_{1}, z_{2}\right)=\left(a\left(z_{1} z_{2}\right) z_{2}^{k}, e^{i \theta} z_{1}^{k} / a\left(z_{1} z_{2}\right)\right)
\end{array}\right.
$$

This finishes the proof in case $\alpha=\beta=(1,1)$.

Now consider the general two-dimensional case $F: D_{\alpha} \mapsto D_{\beta}$. Note that we have the following proper holomorphic mapping:

$$
G\left(z_{1}, z_{2}\right):=\left(F_{1}^{\beta_{1}}\left(z_{1}^{\alpha_{2}}, z_{2}^{\alpha_{1}}\right), F_{2}^{\beta_{2}}\left(z_{1}^{\alpha_{2}}, z_{2}^{\alpha_{1}}\right)\right): D_{(1,1)} \mapsto D_{(1,1)} .
$$

This, together with (5) gives us that

$$
\left\{\begin{array}{l}
F_{1}^{\beta_{1}}\left(z_{1}^{\alpha_{2}}, z_{2}^{\alpha_{1}}\right)=a\left(z_{1} z_{2}\right) z_{1}^{k}, F_{2}^{\beta_{2}}\left(z_{1}^{\alpha_{2}}, z_{2}^{\alpha_{1}}\right)=e^{i \theta} z_{2}^{k} / a\left(z_{1} z_{2}\right) \quad \text { or } \\
F_{1}^{\beta_{1}}\left(z_{1}^{\alpha_{2}}, z_{2}^{\alpha_{1}}\right)=a\left(z_{1} z_{2}\right) z_{2}^{k}, F_{2}^{\beta_{2}}\left(z_{1}^{\alpha_{2}}, z_{2}^{\alpha_{1}}\right)=e^{i \theta} z_{1}^{k} / a\left(z_{1} z_{2}\right) .
\end{array}\right.
$$

Note that if we denote $1^{1 / \alpha_{2}}:=\left\{\mu_{j}: j=0, \ldots, \alpha_{2}-1\right\}, 1^{1 / \alpha_{1}}:=\left\{v_{j}: j=0, \ldots\right.$, $\left.\alpha_{1}-1\right\}, 1^{1 /\left(\alpha_{1} \alpha_{2}\right)}:=\left\{\varepsilon_{j}: j=0, \ldots, \alpha_{1} \alpha_{2}-1\right\}$ then

$$
G\left(\mu_{r} z_{1}, v_{s} z_{2}\right)=G\left(z_{1}, z_{2}\right) \text { for any } z \in D_{\alpha} \text { and } r, s,
$$

which implies that (we consider only the first of two possible forms of $G$, the other form may be treated in exactly the same way)

$$
a\left(z_{1} z_{2}\right)=a\left(\mu_{r} v_{s} z_{1} z_{2}\right) \mu_{r}^{k}, \quad a\left(z_{1} z_{2}\right)=\frac{a\left(\mu_{r} v_{s} z_{1} z_{2}\right)}{v_{s}^{k}}, \quad \text { for all possible } r, s .
$$

Consequently,

$$
\alpha_{1} \alpha_{2} \text { divides } k \text { and } a\left(\varepsilon_{j} \lambda\right)=a(\lambda) \text { for any } \lambda \in E \text {. }
$$

Therefore, the coefficients $c_{l} \neq 0$ of the Taylor expansion of $a$ at 0 are such that $\alpha_{1} \alpha_{2}$ divides $l$. From these pieces of information we easily conclude that $F$ is of the desired form.

Proof of Theorem 5. First note that Theorem 1 implies that both $\alpha$ and $\beta$ are either of rational or of irrational type.

Below we prove that

$$
F\left(V_{0}\right)=V_{0}
$$


If $\alpha$ and $\beta$ are both of rational type, then (6) is a consequence of Lemma 7 and the analytic structure of the set $V_{0}$, which must be preserved under biholomorphic mappings ( $k$ must be 1 and $\lambda_{1}$ must be 0 ).

Now we prove (6) if both $\alpha$ and $\beta$ are of irrational type. Suppose that it does not hold, then it is sufficient to exclude the case when for some $w \in V_{0}$ we have that $F(w) \notin V_{0}$. In that case (use the formula for the Green function from Theorem 1)

$$
-\infty \not \equiv g_{D_{\alpha}}(w ; \cdot)=g_{D_{\beta}}(F(w) ; F(\cdot)) \equiv-\infty,
$$

which is an obvious contradiction.

We know that $V_{0}:=\bigcup_{J=1}^{n} V_{J}$, where $V_{k}:=C^{k-1} \times\{0\} \times C^{n-k}, k=1, \ldots, n$. $V_{0}$ is an analytic set. Because the multiplicity of points of the analytic sets is preserved (6) implies that there is a permutation $\sigma$ such that

$$
F\left(V_{k}\right)=V_{\sigma(k)}
$$

In particular, we get $F(0)=0$, so

$$
\log \left(\left|z_{1}\right|^{\alpha_{1}} \cdots\left|z_{n}\right|^{\alpha_{n}}\right)^{1 /\left(\alpha_{1}+\cdots+\alpha_{n}\right)}=\log \left(\left|F_{1}(z)\right|^{\beta_{1}} \cdots\left|F_{n}(z)\right|^{\beta_{n}}\right)^{1 /\left(\beta_{1}+\cdots+\beta_{n}\right)} .
$$

The formula for the Green function with one pole gives the following

$$
\begin{aligned}
\log \left(\left|z_{1}\right|^{\alpha_{1}} \cdots\left|z_{n}\right|^{\alpha_{n}}\right)^{1 / \alpha_{j}} & =g_{D_{\alpha}}\left(e_{j} ; z\right)=g_{D_{\beta}}\left(s_{j} e_{\sigma(j)} ; F(z)\right) \\
& =\log \left(\left|F_{1}(z)\right|^{\beta_{1}} \cdots\left|F_{n}(z)\right|^{\beta_{n}}\right)^{1 / \beta_{\sigma(J)}}, s_{J} \neq 0, j=1, \ldots, n,
\end{aligned}
$$

where $e_{j}=(0, \ldots, 1, \ldots, 0)$. The last equalities together with (7) give the following:

$$
\frac{\alpha_{1}+\cdots+\alpha_{n}}{\beta_{1}+\cdots+\beta_{n}}=\frac{\alpha_{j}}{\beta_{\sigma(j)}}
$$

from which we easily finish the proof.

Remark 8. The technique used in the proof of Theorem 4 cannot be used in higher dimension. Nevertheless, Theorem 2 combined with Theorem 3 imply that in arbitrary dimension if a proper holomorphic mapping between $D_{\alpha}$ and $D_{\beta}$ exists, then $D_{\alpha}$ and $D_{\beta}$ must be of the same type (either rational or irrational).

\section{REFERENCES}

[Edi-Zwo] A. Edigarian AND W ZwoneK, Invariance of the pluricomplex Green function with many poles under proper mappings with applications, Complex Variables Theory Appl., 35 (1998), 367-380.

[Jar-Pfl] M. JARnicki AND P Pflug, Invariant Distances and Metrics in Complex Analysis, Walter de Gruyter, 1993.

[Kli] M. KLIMEK, Pluripotentıal Theory, Oxford Unıversity Press, 1991.

[Lar-Sig] F LÁRusson AND R. SigurDSSON, Plurisubharmonıc functions and analytic discs on manifolds, J. Reine Angew. Math., 501 (1998), 1-39. 
[Lel] P Lelong, Fonction de Green plunicomplexe et lemmes de Schwarz dans les espaces de Banach, J. Math. Pures Appl., 68 (1989), 319-347

[Pfl-Zwo] P Pflug AND W ZwoneK, Effective formulas for invariant functions-case of elementary Reinhardt domains, Ann. Polon. Math., 69 (1998), 175-196.

[Shi1] S. SHimizu, Holomorphic equivalence problem for a certain class of unbounded Reinhardt domains in $C^{2}$, Osaka J. Math, 28 (1991), 609-621.

[Shi2] S. SHImizu, Holomorphic equivalence problem for a certain class of unbounded Reinhardt domains in $\boldsymbol{C}^{2}$, II, Koda1 Math. J., 15 (1992), 430-444.

\author{
INSTYTUT MATEMATYKI \\ UNIWERSYTET JAGIELLOŃSKI \\ REYMONTA 4, 30-059 KRAKóW \\ Poland \\ E-mail: edigarıaim.uj.edu.pl \\ zwonek@im.uj.edu.pl
}

